\title{
EGFR mutation in squamous cell carcinoma of the lung: does it carry the same connotation as in adenocarcinomas?
}

This article was published in the following Dove Press journal:

OncoTargets and Therapy

28 March 2017

Number of times this article has been viewed

\author{
Amit Joshi' \\ Saurabh Zanwar' \\ Vanita Noronha' \\ Vijay M Patil' \\ Anuradha Chougule ${ }^{2}$ \\ Rajiv Kumar ${ }^{3}$ \\ Amit Janu ${ }^{4}$ \\ Abhishek Mahajan ${ }^{4}$ \\ Akhil Kapoor \\ Kumar Prabhash' \\ 'Department of Medical Oncology, \\ Tata Memorial Hospital, ${ }^{2}$ Molecular \\ Laboratory, Department of Medical \\ Oncology, ${ }^{3}$ Department of \\ Pathology, ${ }^{4}$ Department of Radiology, \\ Tata Memorial Hospital, Mumbai, \\ Maharashtra, India
}

Background: EGFR tyrosine kinase inhibitors (TKIs) have greatly improved the outcomes of $E G F R$ mutation-positive adenocarcinomas of the lung. In contrast, the significance of EGFR mutation in metastatic squamous cell carcinoma (SCC) of the lung has been debated.

Methods: All patients with metastatic SCC who underwent EGFR mutation testing at our center from 2010 to 2015 were included for analysis. EGFR kinase domain mutations were tested using Taqman-based real-time polymerase chain reaction (PCR). Response assessment was done using Response Evaluation Criteria In Solid Tumors (RECIST) 1.1. Kaplan-Meier method was used for calculating progression-free survival (PFS) and overall survival (OS).

Results: EGFR mutation was detected in 29 out of 639 patients with SCC. Furthermore, 19 out of the 29 patients received TKIs at some point during their treatment. TKI therapy led to a partial response in 5 out of 19 patients and stable disease in 4 out of 19 patients. The median PFS of patients treated with TKIs was 5.0 months. The median OS of the whole EGFR-positive SCC cohort was 6.6 months. On univariate analysis, patients having received TKI therapy was the only factor associated with a significantly better OS of 13.48 months versus 2.58 months $(P=0.000)$. On multivariate analysis, patients receiving TKI therapy, Eastern Cooperative Oncology Group-Performance Scale (ECOG-PS) score <2, EGFR exon 19 mutation and nonsmoking status were associated with significantly better OS.

Conclusion: EGFR mutation in SCC of the lung predicts a better outcome if the patient is given TKI, but it may be inferior to the outcomes seen in EGFR-positive adenocarcinomas treated with TKI.

Keywords: EGFR mutation, squamous cell carcinoma, SCC lung, TKI efficacy

\section{Background}

The use of EGFR tyrosine kinase inhibitors (TKIs) ushered in a new era in the treatment of lung cancer, greatly improving the outcomes of EGFR mutation-positive adenocarcinomas (ADCs). ${ }^{1-4}$ The efficacy and benefit of EGFR TKIs have been established beyond doubt in EGFR mutation-positive ADCs, and now there are far more efficacious third-generation TKIs for ADCs progressing on first-generation TKIs. ${ }^{5,6}$ The efficacy of TKIs, however, has not been well studied in EGFR mutation-positive squamous cell carcinomas (SCCs) of the lung.

Performing EGFR testing in SCC is a matter of debate, with no uniform consensus, and guidelines vary for different organizations. ${ }^{7-9}$ Various small series, predominantly from Eastern Asia, have reported the incidence of EGFR kinase mutations in SCC to be in the range of $2 \%-10 \%$, with the caveat that some of these studies have included adenosquamous carcinomas as well in their reports. ${ }^{10-14}$ This is much lower as compared
Correspondence: Kumar Prabhash Department of Medical Oncology, Tata Memorial Hospital, Dr E Borges Road, Parel, Mumbai 400012

Maharashtra, India

Tel+9l 2224177214

Email kprabhashI@gmail.com hereby accept the Terms. Non-commercial uses of the work are permitted without any furcher permission from Dove Medical Press Limited, provided the work is properly attributed. For permission for commercial use of this work, please see paragraphs 4.2 and 5 of our Terms (https://www.dovepress.com/terms.php). 
to the $30 \%-50 \%$ EGFR mutation positivity seen in Asian ADC populations. ${ }^{15-17}$

The clinical significance of EGFR mutations in SCC continues to be an area of research. The lack of robust literature and the rarity of the condition make randomized controlled trials testing TKIs versus chemotherapy for EGFR mutation-positive SCCs an unlikely possibility, and retrospective reports remain the main source of data in this situation.

In our study, we looked at the incidence and clinical significance of EGFR mutations in SCC of the lung in patients treated at our center.

\section{Materials and methods Patient selection}

We analyzed 639 consecutive patients with SCC of the lung diagnosed at our center between January 2010 and December 2015 on whom EGFR mutation analysis was performed. The patients were identified from the prospectively maintained database in the Department of Medical Oncology of the Thoracic Disease Management Group, as part of a study. Diagnosis of SCC was made on the basis of morphology and was supplemented by immunohistochemistry. Adenosquamous carcinomas were excluded from the analysis. Basic demographic information, smoking status, stage at presentation, EGFR mutation type and treatment details were obtained from medical records. Response assessment was done using computed tomography (CT) scan of the thorax and abdomen every 2-4 months. Response to TKI was assessed using Response Evaluation Criteria In Solid Tumors (RECIST) v1.1 criteria. Progression of disease on scan, clinical deterioration or death from any cause, if there was no progression of disease, was defined as progression, for calculation of progression-free survival (PFS). Objective response rate (ORR) was defined as achievement of either a complete or partial response. Clinical benefit rate (CBR) was defined as having stable disease at 4 months from starting TKI therapy. Overall survival (OS) was calculated from the date of diagnosis as advanced-stage disease, to the date of death. PFS with TKI therapy and OS data for the whole cohort were obtained.

\section{Ethical approval}

All procedures performed in studies involving human participants were in accordance with the ethical standards of the institutional and/or national research committee and with the 1964 Declaration of Helsinki and its later amendments or comparable ethical standards.

\section{Informed consent}

This was a retrospective study approved by the institutional ethics committee attached to the Tata Memorial Hospital, Mumbai (project number 1043). Waiver of consent was obtained from the institutional review board in view of the retrospective nature of the study.

\section{Mutation analysis}

EGFR mutation testing was performed on DNA extracted from formalin-fixed paraffin-embedded (FFPE) blocks. This has been reported in our previous paper. ${ }^{18}$

\section{Statistical analysis}

PFS with TKI therapy was calculated from the date of starting TKI till progression or the last follow-up. OS for the whole cohort was calculated from the date of diagnosis till death or the last follow-up. Follow-up data were censored on December 31, 2015. The Kaplan-Meier method was used for calculating PFS and OS. Log-rank test was performed for identifying factors affecting OS of the whole cohort, and multivariate analysis was performed using Cox regression. SPSS 20.0 software was used for statistical analysis.

\section{Results}

EGFR mutation was detected in 4.5\% (29 out of 639) of patients with SCC tested for EGFR mutation. Median age of the patients in the EGFR-positive cohort was 59 years (range: 37-80 years), with 22 males and 7 females. Exon 19 was the most common mutation site, seen in 15 out of 29 patients, followed by mutation in exon 21 in 13 out of 29 patients. Approximately 65\% (19 out of 29) of patients received TKI at some point of time during their treatment course. The TKI used was erlotinib in 10 patients and gefitinib in 9 patients. None of the 19 patients had received afatinib. Seven out of 19 patients with TKI therapy had received TKI as frontline therapy based on the treating physician's judgment of the patient being unfit for platinum-based doublet therapy. History of cigarette smoking was available in 24 patients, and out of these, 10 were cigarette smokers with a median smoking index of 37 pack-years. Moreover, 14 out of 29 patients had a performance status of $\geq 2$ on the Eastern Cooperative Oncology Group-Performance Scale (ECOG-PS). Data on performance status were missing for 3 patients. The median number of lines of systemic therapy received for the whole cohort was 1 (range: $0-5$ ). The baseline characteristics of the cohort are shown in Table 1.

Response assessment for patients receiving TKI showed partial response in 5 out of 19 patients, stable disease in 4 out 
Table I Demographic and other baseline characteristics

\begin{tabular}{ll}
\hline Characteristics & Findings \\
\hline Median age, years & 59 years (range: 37-80) \\
Sex & \\
$\quad$ Male, $\mathrm{n}$ & 22 \\
$\quad$ Female, $\mathrm{n}$ & 7 \\
Smokers, $\mathrm{n}$ & $10 / 29 *(34.4 \%)$ \\
EGFR mutation, $\mathrm{n}$ & \\
Exon 19 & $15(51.7 \%)$ \\
Exon 20 & $1(3.4 \%)$ \\
Exon 2I & $13(44.8 \%)$ \\
TKI therapy at some point during treatment & $19 / 29(65.5 \%)$ \\
TKI as first-line therapy & $7 / 29(24.1 \%)$ \\
ECOG-PS score & \\
0 & 2 \\
I & 10 \\
2 & 10 \\
$>2$ & 4 \\
Data not available & 3 \\
\hline
\end{tabular}

Notes: EGFR mutation-positive $\mathrm{SCC}$ of the lung: $\mathrm{n}=29$. *Details of smoking history were available in only 24 out of the 29 patients.

Abbreviations: ECOG-PS, Eastern Cooperative Oncology Group-Performance Scale; SCC, squamous cell carcinoma; TKI, tyrosine kinase inhibitor.

of 19 patients and progression on first evaluation in 3 patients. Formal response assessment was not available for 6 patients due to loss to follow-up. They were considered to have progressed for the purpose of analysis. This led to an ORR of $26.3 \%$ ( $95 \%$ confidence interval [CI]: $11.8 \%-484.8 \%$ ) and CBR of $47.3 \%$ (95\% CI: $24.8 \%-69.7 \%$ ). None of the 19 patients achieved a complete response to TKI. The response rates are shown in Table 2.

The median PFS for patients on TKI, irrespective of the number of previous lines of treatment, was 5.0 months ( $95 \%$ CI: 1.8-8.2 months) (Figure 1). The median OS of the whole EGFR mutation-positive SCC cohort was 6.6 months (95\% CI: 0-14.1 months). On univariate analysis, patients having received TKI was the only factor associated with a significantly better OS of 13.48 months (95\% CI: 7.4-19.4 months) versus 2.58 months ( $95 \%$ CI: $0.7-5.0$ months) in patients not exposed to TKI $(P=0.000)$ (Figure 2). Patients with exon 19 mutation tended to have better OS $(P=0.059)$ on univariate

Table 2 Response rate for TKI therapy

\begin{tabular}{ll}
\hline Response to TKI & $\%(\mathbf{n})$ \\
\hline CR & 0 \\
PR & $26.3(5 / 19)$ \\
SD & $21(4 / 19)$ \\
PD & $15.8(3 / 19)$ \\
CBR (CR + PR + SD at 4 months) & $47.3(9 / 19)$ \\
Data not available* & $36.8(7 / 19)$ \\
\hline
\end{tabular}

Note: *Considered as progression for analysis purpose.

Abbreviations: $C B R$, clinical benefit rate; $C R$, complete response; $P D$, progressive disease; PR, partial response; SD, stable disease; TKI, tyrosine kinase inhibitor.

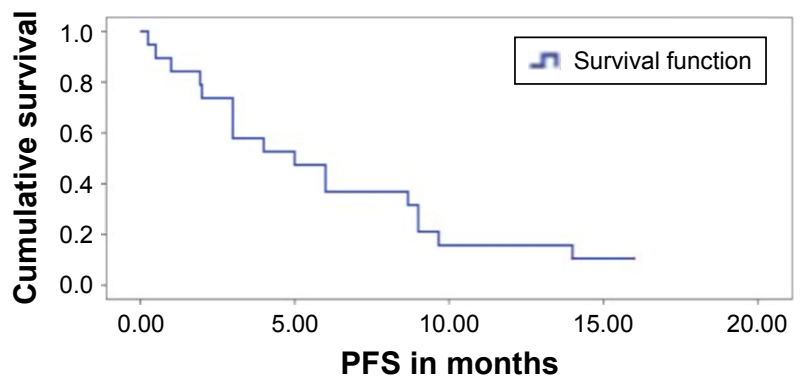

Figure I Progression-free survival (PFS) of whole cohort.

analysis. On multivariate analysis using Cox regression, patients receiving TKI and having exon 19 mutation were associated with significant improvement in survival. ECOG-PS score $>1$ and history of smoking had a negative impact on survival. The results of multivariate analysis are shown in Table 3.

\section{Discussion}

EGFR mutation, as a biomarker for response to TKI in SCC of the lung has limited data. The remarkable improvement in the outcomes of EGFR mutation-positive ADC with the use of TKI has generated a lot of interest for the possibility of a similar impact of TKI in EGFR-mutant SCC. The available literature so far has failed to attest the same. This study was conceived in view of the conflicting reports of responses to TKI in this patient population, and with most reports being from Eastern Asian populations, the applicability of the results to other populations was questionable. In addition, many of these reports had included adenosquamous carcinomas as well. To avoid ambiguity, we have excluded adenosquamous carcinomas from the study population. To the best of our knowledge, this study represents one of the

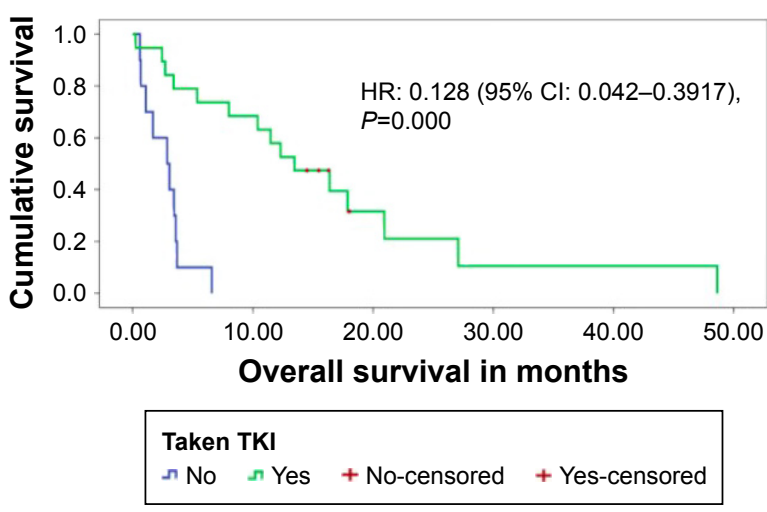

Figure 2 Overall survival for patients who had taken TKI versus those who had not been given TKI therapy.

Abbreviations: $\mathrm{Cl}$, confidence interval; HR, hazard ratio; TKI, tyrosine kinase inhibitor. 
Table 3 Multivariate analysis for factors affecting overall survival

\begin{tabular}{lllll}
\hline Variable & P-value & HR & \multicolumn{2}{c}{$\mathbf{9 5 \%} \mathbf{C l}$ for $\mathbf{H R}$} \\
\cline { 4 - 5 } & & & Lower & Upper \\
\hline Age $<60$ years & 0.950 & 1.002 & 0.955 & $1.05 \mathrm{I}$ \\
Exon 19 mutation & 0.024 & 0.226 & 0.062 & 0.820 \\
Smoking history & 0.013 & 4.994 & 1.407 & 17.720 \\
ECOG-PS $>$ I & 0.001 & 17.775 & 3.054 & 103.466 \\
Treatment with a TKI & 0.000 & 0.036 & 0.007 & 0.193 \\
\hline
\end{tabular}

Abbreviations: $\mathrm{Cl}$, confidence interval; ECOG-PS, Eastern Cooperative Oncology Group-Performance Scale; HR, hazard ratio; TKI, tyrosine kinase inhibitor.

largest single-center studies of SCC of the lung with an EGFR mutation.

The baseline characteristics were comparable to those of ADC previously reported from our center, except for a higher percentage of patients being males and smokers. Our study showed that objective responses are seen with TKI in mutation-positive patients in approximately one-quarter of the patients, which is similar to a previous report by Shukuya et al. ${ }^{19}$ The ORR, however, was much lower compared to our EGFR mutation-positive ADC, whereby data from multiple studies of EGFR mutation-positive ADCs have shown excellent response rates in the range of $\sim 65 \%-75 \%{ }^{20}$ The median PFS in SCC patients treated with TKI was 5 months in our study, which was again lower compared to the usual PFS of 8-12 months seen in ADC treated with TKI. ${ }^{1,21,22}$ One factor that could have led to this finding was that the majority of the patients had not received TKI upfront during the treatment course.

The median OS of a previously reported $E G F R$ mutation-positive ADC historical control from our center was $\sim 21$ months (95\% CI: 12.4-25.6 months). ${ }^{20}$ This appears to be much longer than the median OS of 13.48 months $(95 \%$ CI: 7.4-19.4 months) for those who received TKI in the current EGFR mutation-positive SCC cohort. The median OS for the whole EGFR mutation-positive SCC cohort in the current study was even lower, at 6.6 months (95\% CI: 0-14.1 months). ECOG-PS score was $>1$ in the majority of the patients in both these studies $(52.2 \%$ in the ADC population versus $48.8 \%$ in the SCC population), which makes it less likely to be a confounding factor for poor OS. This difference in OS is in contrast to the previous report by Cho et al, ${ }^{10}$ wherein they found comparable median survivals in EGFR mutation-positive ADC and non-ADC groups.

Overall, these findings suggest that TKI may have activity in EGFR-positive SCC, but the effect appears to be somewhat lower than that seen in ADCs. Various hypotheses have been put forward for the same, including the presence of other "nonmutually exclusive" mutations such as PIK3CA, which are more frequently seen in SCCs than in ADCs and may be responsible for the lower responses. It would be interesting to search for other associated mutations in the tumor tissue. ${ }^{23-25}$ In our study, use of TKI therapy at any point of time during treatment, presence of exon 19 mutation, nonsmoking status and ECOG-PS score $<2$ were independent predictors of survival. These are generally consistent with the previous data from ADC patients. ${ }^{1,2,4}$ The interpretation of the results of this analysis is limited by the small sample size. Moreover, a direct comparison between the SCC and the historical ADC cohort is not the ideal way to draw a conclusion and is associated with limitations.

The prognosis of metastatic SCC of the lung has improved with the recent approval of immunotherapy. ${ }^{26}$ Newer treatment strategies, especially addressing the currently limited utility of targeted therapy in SCC of lung, are required. Search for associated mutations probably governing the lower response rates represents a promising future research strategy. Furthermore, one way to improve the outcomes of EGFR mutation-positive SCC of the lung being treated with TKIs could be to predict the development of resistance to EGFR therapy by liquid biopsy with serial circulating free DNA (cfDNA) measurement. ${ }^{27}$ This can help to detect resistance to EGFR TKIs early and tailor therapy accordingly.

\section{Conclusion}

Treatment with TKI in EGFR-mutated SCC of the lung is associated with improvement in survival, although the outcomes appear to be somewhat inferior compared to that of EGFR mutation-positive ADCs treated with TKIs.

\section{Acknowledgments}

We would like to thank Dr Prabhat Bhargava for his help in data collection and $\mathrm{Mr}$ Amol Bansode for his help in statistical analysis. There was no funding allocated for the study. This study, in its abstract form, was selected as a poster at the European Society for Medical Oncology (ESMO) 2016.

\section{Disclosure}

The authors report no conflicts of interest in this work.

\section{References}

1. Zhou C, Wu YL, Chen G, et al. Final overall survival results from a randomised, phase III study of erlotinib versus chemotherapy as firstline treatment of EGFR mutation-positive advanced non-small-cell lung cancer (OPTIMAL, CTONG-0802). Ann Oncol. 2015;26(9): $1877-1883$. 
2. Schuette W, Schirmacher P, Eberhardt WE, et al. EGFR mutation status and first-line treatment in patients with stage III/IV non-small cell lung cancer in Germany: an observational study. Cancer Epidemiol Biomarkers Prev. 2015;24(8):1254-1261.

3. Venugopalan A, Lee MJ, Niu G, et al. EGFR-targeted therapy results in dramatic early lung tumor regression accompanied by imaging response and immune infiltration in EGFR mutant transgenic mouse models. Oncotarget. 2016;7(34):54137-54156.

4. Li N, Yang L, Ou W, Zhang L, Zhang SL, Wang SY. Meta-analysis of EGFR tyrosine kinase inhibitors compared with chemotherapy as second-line treatment in pretreated advanced non-small cell lung cancer. PLoS One. 2014;9(7):e102777.

5. Wang S, Cang S, Liu D. Third-generation inhibitors targeting EGFR T790M mutation in advanced non-small cell lung cancer. J Hematol Oncol. 2016;9:34.

6. Wang S, Tsui ST, Liu C, Song Y, Liu D. EGFR C797S mutation mediates resistance to third-generation inhibitors in T790M-positive non-small cell lung cancer. J Hematol Oncol. 2016;9(1):59.

7. Keedy VL, Temin S, Somerfield MR, et al. American Society of Clinical Oncology provisional clinical opinion: epidermal growth factor receptor (EGFR) mutation testing for patients with advanced non-small-cell lung cancer considering first-line EGFR tyrosine kinase inhibitor therapy. J Clin Oncol. 2011;29(15):2121-2127.

8. Felip E, Gridelli C, Baas P, Rosell R, Stahel R; Panel Members. Metastatic non-small-cell lung cancer: consensus on pathology and molecular tests, first-line, second-line, and third-line therapy: 1st ESMO consensus conference in lung cancer; Lugano 2010. Ann Oncol. 2011; 22(7):1507-1519.

9. Lindeman NI, Cagle PT, Beasley MB, et al. Molecular testing guideline for selection of lung cancer patients for EGFR and ALK tyrosine kinase inhibitors: guideline from the College of American Pathologists, International Association for the Study of Lung Cancer, and Association for Molecular Pathology. Arch Pathol Lab Med. 2013;137(6):828-860.

10. Cho SH, Park LC, Ji JH, et al. Efficacy of EGFR tyrosine kinase inhibitors for non-adenocarcinoma NSCLC patients with EGFR mutation. Cancer Chemother Pharmacol. 2012;70(2):315-320.

11. Miyamae Y, Shimizu K, Hirato J, et al. Significance of epidermal growth factor receptor gene mutations in squamous cell lung carcinoma. Oncol Rep. 2011;25(4):921-928.

12. Fang W, Zhang J, Liang W, et al. Efficacy of epidermal growth factor receptor-tyrosine kinase inhibitors for Chinese patients with squamous cell carcinoma of lung harboring EGFR mutation. J Thorac Dis. 2013; 5(5):585-592.

13. Xu J, Zhang Y, Jin B, et al. Efficacy of EGFR tyrosine kinase inhibitors for non-adenocarcinoma lung cancer patients harboring EGFRsensitizing mutations in China. J Cancer Res Clin Oncol. 2016;142(6): 1325-1330.

14. Wu YL, Zhong WZ, Li LY, et al. Epidermal growth factor receptor mutations and their correlation with gefitinib therapy in patients with non-small cell lung cancer: a meta-analysis based on updated individual patient data from six medical centers in mainland China. J Thorac Oncol. 2007;2(5):430-439.
15. Shi Y, Au JS, Thongprasert S, et al. A prospective, molecular epidemiology study of EGFR mutations in Asian patients with advanced non-small cell lung cancer of adenocarcinoma histology (PIONEER). J Thorac Oncol. 2014;9(2):154-162.

16. Hosokawa S, Toyooka S, Fujiwara Y, et al. Comprehensive analysis of EGFR signaling pathways in Japanese patients with non-small cell lung cancer. Lung Cancer. 2009;66(1):107-113.

17. Midha A, Dearden S, McCormack R. EGFR mutation incidence in non-small-cell lung cancer of adenocarcinoma histology: a systematic review and global map by ethnicity (mutMapII). Am J Cancer Res. 2015; 5(9):2892-2911.

18. Chougule A, Prabhash K, Noronha V, et al. Frequency of EGFR mutations in 907 lung adenocarcioma patients of Indian ethnicity. PLoS One. 2013;8(10):e76164.

19. Shukuya T, Takahashi T, Kaira R, et al. Efficacy of gefitinib for nonadenocarcinoma non-small-cell lung cancer patients harboring epidermal growth factor receptor mutations: a pooled analysis of published reports. Cancer Sci. 2011;102(5):1032-1037.

20. Noronha V, Prabhash K, Thavamani A, et al. EGFR mutations in Indian lung cancer patients: clinical correlation and outcome to EGFR targeted therapy. PLoS One. 2013;8(4):e61561.

21. Maemondo M, Inoue A, Kobayashi K, et al. Gefitinib or chemotherapy for non-small-cell lung cancer with mutated EGFR. $N$ Engl J Med. 2010;362(25):2380-2388

22. Rosell R, Carcereny E, Gervais R, et al; Spanish Lung Cancer Group in collaboration with Groupe Français de Pneumo-Cancérologie and Associazione Italiana OncologiaToracica. Erlotinib versus standard chemotherapy as first-line treatment for European patients with advanced EGFR mutation-positive non-small-cell lung cancer (EURTAC): a multicentre, open-label, randomised phase 3 trial. Lancet Oncol. 2012;13(3):239-246.

23. Kawano O, Sasaki H, Endo K, et al. PIK3CA mutation status in Japanese lung cancer patients. Lung Cancer. 2006;54(2):209-215.

24. Hammerman PS, Janne PA, Johnson BE. Resistance to epidermal growth factor receptor tyrosine kinase inhibitors in non-small cell lung cancer. Clin Cancer Res. 2009;15:7502-7509.

25. Lee SY, Jin G, Choi JE, et al. Somatic mutations in epidermal growth factor receptor signaling pathway genes in non-small cell lung cancers J Thorac Oncol. 2010;5(11):1734-1740.

26. Ma W, Gilligan BM, Yuan J, Li T. Current status and perspectives in translational biomarker research for PD-1/PD-L1 immune checkpoint blockade therapy. J Hematol Oncol. 2016;9(1):47.

27. Zhou Q, Yang J-J, Chen Z-H, et al. Serial cfDNA assessment of response and resistance to EGFR-TKI for patients with EGFR-L858R mutant lung cancer from a prospective clinical trial. J Hematol Oncol. 2016;9(1):86.
OncoTargets and Therapy

\section{Publish your work in this journal}

OncoTargets and Therapy is an international, peer-reviewed, open access journal focusing on the pathological basis of all cancers, potential targets for therapy and treatment protocols employed to improve the management of cancer patients. The journal also focuses on the impact of management programs and new therapeutic agents and protocols on

\section{Dovepress}

patient perspectives such as quality of life, adherence and satisfaction. The manuscript management system is completely online and includes a very quick and fair peer-review system, which is all easy to use. Visit http://www.dovepress.com/testimonials.php to read real quotes from published authors. 